\title{
The effects of information redundancy on evaluations of social stimuli
}

\author{
ROBERT S. WYER, JR., University of Mlinois, Chicago Circle, \\ Chicago, Ill. 60680
}

Subjects were asked to estimate their liking for persons described by pairs of adjectives. These estimates were analyzed as a function of the favorableness of the adjectives in each pair and the redundancy of these adjectives. The degree of redundancy of an adjective $B$ with a second adjective $A$ was defined as the conditional probability of occurrence of $B$ given $A$; these probabilities were estimated from normative data. Results supported the hypothesis that if adjectives are redundant with others presented, they have less effect upon evaluations. This study, which led to conclusions similar to those made by Dustin \& Baldwin (1966), eliminated several methodological difficulties in the earlier research.

Critical tests of the manner in which information about a person combines to affect evaluations of that person may need to take into account the degree of redundancy in the information presented. Two pieces of information are redundant if one can be inferred from the other with high probability. Dustin \& Baldwin (1966) found support for the hypothesis that evaluations based upon pairs of highly redundant adjectives were less polarized (relative to the mean rating of the single adjectives) than were evaluations based upon less redundant pairs. However, their study is difficult to interpret for several reasons. First, only a small number of adjectives was considered. Second, their hypothesis was strongly supported only when the adjectives presented were unfavorable. Finally, the degree of redundancy of the adjectives involved appeared to be correlated with the mean evaluation of the adjectives presented; that is, the mean rating of highly redundant adjectives presented separately was generally more extreme than the mean rating of less redundant adjectives presented separately. Therefore, the relatively small difference between evaluations based upon a pair of highly redundant adjectives and the mean of the evaluations based upon each of the two adjectives in isolation could be due primarily to "ceiling" effects at the extremes of the rating scale used to record judgments.

The present study attempted to test the hypothesis suggested by Dustin and Baldwin using a procedure that would eliminate these criticisms and would have more general applications. \section{METHOD}

Collection of Normative Data

The favorableness and redundancy of the adjectives presented were each defined on the basis of normative data. To obtain these data, a set of 240 adjectives was selected, the majority of which were chosen from the set of 555 compiled by Anderson (1968). To obtain favorableness norms, 75 introductory psychology students were asked to estimate, on a scale from -10 (very unfavorable) to +10 (very favorable), how favorable they believed each of the adjectives to be. The mean rating of each adjective was used as its normative value.

In this study, the redundancy of one adjective (B) with another(A) was assumed to be a function of the conditional probability of occurrence of the first given the second $\left(\mathrm{P}_{\mathrm{B} / \mathrm{A}}\right)$. To obtain data for calculating these probabilities, a set of 40 stimulus persons was selected. These persons were assumed to vary greatly in likeableness and to be representative of the population of persons with whom Ss were familiar (e.g., Josef Stalin, John Kennedy, "your high school English teacher," "the person you have met most recently," et al). Each of 225 Ss was given two of these stimulus persons and asked to indicate which of the 240 adjectives under consideration would describe each. For the relative frequencies with which each adjective was assigned to the 450 persons rated, it was possible to calculate the conditional probability that a person described by any particular adjective was also described by each of the other adjectives. These data were assumed to reflect the degree of association between these traits in the population from which $\mathrm{Ss}$ in this study were drawn.
Preparation of Materials

Adjectives were selected at five favorableness levels: $\mathrm{H}$ (means ranging from +5 to +7$), M+(+2$ to +4$), O(-1$ to +1$), M-(-4$ to -2$)$ and $L(-7$ to $-5)$. From these adjectives, 120 pairs were constructed as follows. First, five adjectives (Set $A_{1}$ ), one at each favorableness level, were chosen. Eight additional adjectives (Set $B_{1}$ ), two at each favorableness level except $M-$, were also selected. (Adjectives at the $M-$ level could not be selected which met the criteria to be described.) The two Set $B_{1}$ adjectives at each level differed in the probability that they were associated with each Set $A_{1}$ adjective by at least .15. Each Set $A_{1}$ adjective was then paired with each Set $B_{1}$ adjective to form 40 different adjective pairs. Two additional stimulus replications were constructed by pairing each Set $A_{1}$ adjective with eight additional (Set $B_{2}$ ) adjectives which met the criteria described above, and by pairing each Set $B_{1}$ adjective with each of five (Set $A_{2}$ ) by pairing each Set $B_{1}$ adjective with each of five (Set $A_{2}$ ) adjectives according to these criteria. Collapsed over replications, the average difference in the probability that high and low redundancy Set $B$ adjectives were associated with the $\mathrm{A}$ adjectives accompanying them was $.25, .35, .30$, and .21 at $\mathrm{H}$, $M+, 0$, and $L$ favorableness levels, respectively.

To obtain data for an additional analysis of the effects of redundancy, each of the 10 adjectives in Sets $A_{1}$ and $A_{2}$ was paired with itself to form 10 maximally redundant pairs. For control purposes, each of these adjectives was also presented in isolation.

Instructions

Thirty-three introductory psychology students were run in groups of eight to nine each. Pairs of adjectives, which were presented to Ss on slides, were ordered randomly. Ss were instructed to form an impression of the person described by each set of adjectives presented, and then to estimate their liking for the person along a 21 -point scale from -10 (would dislike very much) to +10 (would like very much).

\section{RESULTS}

It was expected that the effect of Set B adjectives on evaluations would be less if they were highly redundant with the Set $A$ adjectives paired with them than if they were less redundant with these adjectives. To test this hypothesis, each S's evaluation of a pair containing a Set $A$ adjective and a Set B adjective associated with it with low probability was subtracted from his evaluation of the corresponding pair containing the same Set $A$ adjective and a Set $B$ adjective associated with it with high probability. If the hypothesis is correct, this difference score should generally be positive when the Set $B$ adjectives in question are unfavorable, and should be negative when the Set $B$ adjectives involved are favorable.

The difference scores described above are shown in Fig. 1 for each stimulus replication as a function of B-adjective favorableness. The main effect of $B$-adjective favorableness, collapsed over replications and A-adjective favorableness, was significant $[F(3,288)=28.90 ; p<.001]$. This variable interacted significantly with replications $(F=6.50 ; p<.01)$; however, analysis of each replication separately yielded a significant main effect of B-adjective favorableness in each case $[F(3,96) \geqslant 2.81$; $p<.05]$. The generally consistent decrease in the difference scores over favorableness levels supports the hypothesis in question. However, the magnitude of the effect depends in part upon idiosyncratic characteristics of the adjectives involved.

As a somewhat more direct test of redundancy effects, a subset of the single adjectives and adjective pairs at each of four favorableness levels $(\mathrm{H}, \mathrm{M}+, \mathrm{O} \& \mathrm{~L})$ were selected from the total group presented. These consisted of eight Set $A$ adjectives, two at each favorableness level, presented in isolation, these same adjectives each presented in a pair with itself (complete redundancy), these adjectives each paired with a second equally favorable adjective associated with it with high probability (high redundancy), and these adjectives each paired with an equally favorable adjective associated with it with low probability (low redundancy). The probability that adjectives in high redundancy pairs were associated was at least .45 greater than the probability 
Table 1

Mean Evaluations of Adjective Sets as a Function of Favorableness Level and Information Redundancy

\begin{tabular}{ccccc}
\hline & & \multicolumn{3}{c}{ Redundancy } \\
\cline { 3 - 5 } $\begin{array}{c}\text { Level of } \\
\text { Favorableness }\end{array}$ & $\begin{array}{c}\text { Single } \\
\text { Adjective }\end{array}$ & $\begin{array}{c}\text { Complete } \\
\text { Redundancy } \\
\text { (Identical) }\end{array}$ & $\begin{array}{c}\text { High } \\
\text { Redundancy }\end{array}$ & $\begin{array}{c}\text { Low } \\
\text { Redundancy }\end{array}$ \\
\hline H & 6.26 & 6.36 & 6.76 & 7.02 \\
M+ & 2.04 & 1.48 & 1.62 & 2.20 \\
O & -.61 & -.97 & -1.09 & -1.24 \\
L & -5.83 & -6.26 & -6.95 & -7.04 \\
\hline
\end{tabular}

that adjectives in corresponding low redundancy pairs were associated.

Table 1 shows mean evaluations of single adjectives and adjective pairs as a function of favorableness level and redundancy level. It was predicted that evaluations would become more extreme as redundancy decreased. The interaction of favorableness and redundancy $[F(9,288)=1.92 ; p<.05]$ supports this hypothesis. At all levels of favorableness, redundancy effects were in the predicted direction. (The decrease in evaluations of pairs of $\mathrm{O}$ adjectives as redundancy decreased is also consistent with the hypothesis since evaluations of adjectives at this level were actually somewhat negative.)

In three of the four cases, ratings were more extreme when based upon two identical adjectives than when based upon only one of these traits. This might not be expected since the adjectives contained in these pairs were completely redundant. Possibly the additional adjective, although identical to the first, increased the subjective probability that the information presented is correct, and consequently increased the extremity of evaluations. A similar interpretation of repetition effects has been offered by L. Anderson \& Fishbein (1965).

\section{DISCUSSION}

The results of this study provide fairly strong confirmation of the hypothesis that pieces of information about an object have less effect upon evaluations of that object if they are redundant with other information presented about it. These results are consistent with findings by Dustin \& Baldwin (1966) but eliminate some ambiguities in the earlier study.

Data in Fig. 1 suggest that the effects of differences in redundancy are greater at extreme levels of favorableness than at more neutral levels. This would be consistent with two alternative interpretations of redundancy effects. One interpretation assumes that a S's evaluation of an object is based only on the attributes specified in the description of the object. If two or more pieces of information are presented, aspects of the information that are redundant are taken into account only once. This formulation would imply that the evaluation of an object $O$ based upon two adjectives $A$ and $B$, assuming that $B$ is presented following $A$, would be a function of the expression

$$
E_{O}=E_{A}+\left(1-P_{B / A}\right) E_{B}
$$

where $E_{A}$ and $E_{B}$ are the evaluations based upon $A$ and $B$ separately and $P_{B / A}$ is the conditional probability of $B$ given $A$.

$A$ second interpretation assumes that evaluations of an object are based not only upon the specific adjectives contained in the description of that person but also upon other attributes, not specifically mentioned, that are inferred with some probability on the basis of these adjectives. To the extent that other attributes are associated with those specified in the description with high probability they are taken into account in the evaluation based upon this description, and subsequent information that the object described actually possesses them has less effect. To clarify this, assume that evaluations are predictable from the expression $E_{o}=\Sigma P_{i} F_{i}$, where $F_{i}$ is the favorableness of the $i^{\text {th }}$ attribute relevant to the evaluation of $O$ and $P_{i}$ is the probability that $O$ possesses the attribute. This formulation is sim-

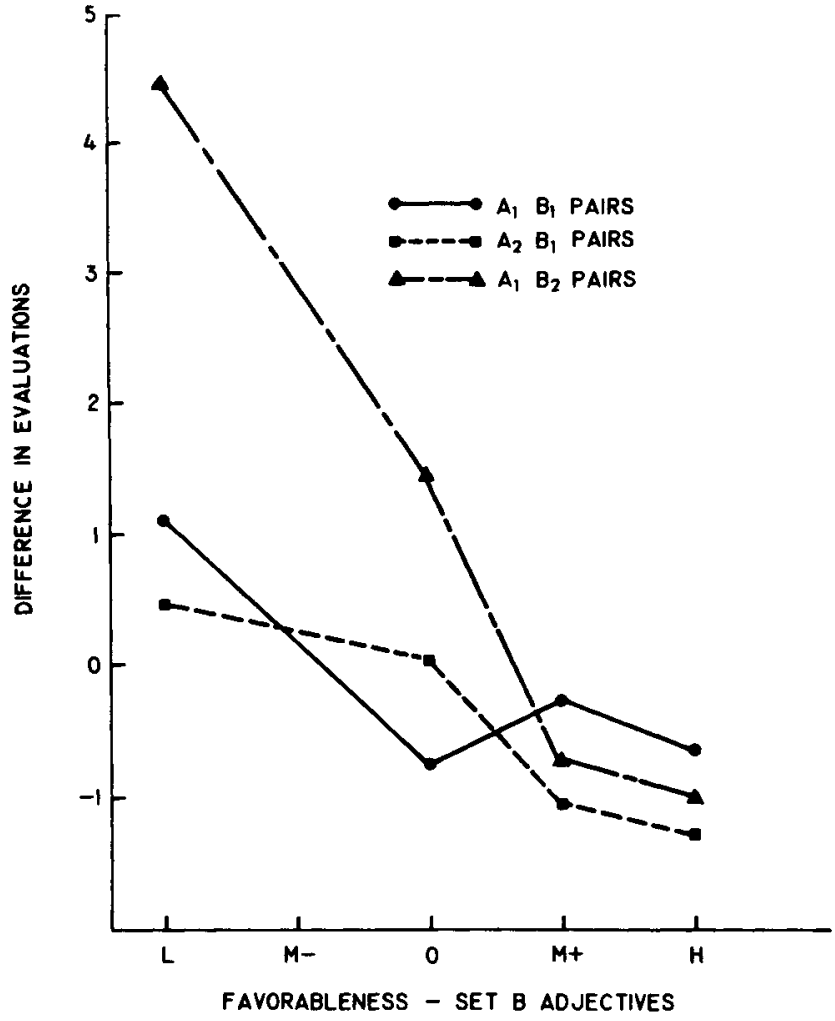

Fig. 1. Difference between evaluations of pairs containing Set B adjectives high in redundancy and evaluations of pairs containing Set $B$ adjectives low in redundancy, as a function of the favorableness of Set B adjectives.

ilar to that proposed by Fishbein (1963). As a further oversimplification, assume that only two attributes, $A$ and $B$, are relevant to the evaluation of $O$. Then, the evaluation based upon a description containing only attribute $A$ would be predicted by the equation $E_{O}=F_{A}+P_{B / A} F_{B}$. (The probability associated with the information presented is assumed to be unity; in actuality, this may not always be the case.) If $B$ is then added to the description, the equation becomes $\mathrm{E}_{\mathrm{O}}=\mathrm{F}_{\mathrm{A}}+\mathrm{F}_{\mathrm{B}}$. The change in evaluations due to the addition of $B$ would therefore be less when $P_{B} / A$ is high. Note that this formulation, unlike the first, makes a distinction between the favorableness of an adjective and the evaluation based upon it. Furthermore, it is clear that since in reality several attributes might typically be inferred with some probability on the basis of the information presenter with some probability on the basis of the information presented, an exact quantitative prediction of redundancy effects would be difficult unless these probabilities can be specified. REFERENCES

ANDERSON, N. H. Likeableness ratings of 555 personality trait adjectives. Journal of Personality \& Social Psychology, 1968, 9, 272-279.

ANDERSON, L., \& FISHBEIN, M. Prediction of attitude from the number, strength and evaluative aspect of beliefs about the attitude object: A comparison of summation and congruity theories. Journal of Personality \& Social Psychology, 1965, 2, 437-43.

DUSTIN, D. S., \& BALDWIN, P. M. Redundancy in impression formation. Journal of Personality \& Social Psychology, 1966, 3 500-506.

FISHBEIN, M. An investigation of the relationship between beliefs about an object and the attitude toward that object. Human Relations, 1963, $16,233-239$.

NOTE

1. This study was supported by National Science Foundation Grant GS 1356. Appreciation is given to Mitchell Kaufman for his assistance in collecting data for the study, and to Norman H. Anderson for his comments on a longer draft of the paper. 